\title{
LINEAR FILTERING OF SYSTEMS WITH MEMORY AND APPLICATION TO FINANCE
}

\author{
A. INOUE, Y. NAKANO, AND V. ANH
}

Received 29 July 2004; Revised 25 March 2005; Accepted 5 April 2005

We study the linear filtering problem for systems driven by continuous Gaussian processes $V^{(1)}$ and $V^{(2)}$ with memory described by two parameters. The processes $V^{(j)}$ have the virtue that they possess stationary increments and simple semimartingale representations simultaneously. They allow for straightforward parameter estimations. After giving the semimartingale representations of $V^{(j)}$ by innovation theory, we derive KalmanBucy-type filtering equations for the systems. We apply the result to the optimal portfolio problem for an investor with partial observations. We illustrate the tractability of the filtering algorithm by numerical implementations.

Copyright (c) 2006 A. Inoue et al. This is an open access article distributed under the Creative Commons Attribution License, which permits unrestricted use, distribution, and reproduction in any medium, provided the original work is properly cited.

\section{Introduction}

Let $T$ be a positive constant. In this paper, we use the following Gaussian process $V=$ $\left(V_{t}, t \in[0, T]\right)$ with stationary increments as the driving noise process:

$$
V_{t}=W_{t}-\int_{0}^{t}\left(\int_{-\infty}^{s} p e^{-(q+p)(s-u)} d W_{u}\right) d s, \quad 0 \leq t \leq T
$$

where $p$ and $q$ are real constants such that

$$
0<q<\infty, \quad-q<p<\infty
$$

and $\left(W_{t}, t \in \mathbb{R}\right)$ is a one-dimensional Brownian motion satisfying $W_{0}=0$. The parameters $p$ and $q$ describe the memory of $V$. In the simplest case $p=0, V$ is reduced to the Brownian motion, that is, $V_{t}=W_{t}, 0 \leq t \leq T$.

It should be noticed that (1.1) is not a semimartingale representation of $V$ with respect to the natural filtration $\mathscr{F}^{V}=\left(\mathscr{F}_{t}^{V}, t \in[0, T]\right)$ of $V$ since $\left(W_{t}, t \in[0, T]\right)$ is not $\mathscr{F}^{V}$-adapted. Using the innovation theory as described by Liptser and Shiryaev [21] and a result by Anh et al. [2], we show (Theorem 2.1) that there exists a one-dimensional 
Brownian motion $B=\left(B_{t}, t \in[0, T]\right)$, called the innovation process, satisfying

$$
\begin{gathered}
\sigma\left(B_{s}, s \in[0, t]\right)=\sigma\left(V_{s}, s \in[0, t]\right), \quad 0 \leq t \leq T, \\
V_{t}=B_{t}-\int_{0}^{t}\left(\int_{0}^{s} l(s, u) d B_{u}\right) d s, \quad 0 \leq t \leq T
\end{gathered}
$$

where $l(t, s)$ is a Volterra kernel given by

$$
l(t, s)=p e^{-(p+q)(t-s)}\left\{1-\frac{2 p q}{(2 q+p)^{2} e^{2 q s}-p^{2}}\right\}, \quad 0 \leq s \leq t \leq T .
$$

With respect to the natural filtration $\mathscr{F}^{B}=\left(\mathscr{F}_{t}^{B}, t \in[0, T]\right)$ of $B$, which is equal to $\mathscr{F} V$, (1.4) gives the semimartingale representation of $V$. Thus the process $V$ has the virtue that it possesses the property of a stationary increment process with memory and a simple semimartingale representation simultaneously. We know no other process with this kind of properties. The two properties of $V$ become a great advantage, for example, in its parameter estimation.

In $[1,2,10]$, the process $V$ is used as the driving process for a financial market model with memory. The empirical study for S\&P 500 data by Anh et al. [3] shows that the model captures reasonably well the memory effect when the market is stable. The work in these references suggests that the process $V$ can serve as an alternative to Brownian motion when the random disturbance exhibits dependence between different observations.

In this paper, we are concerned with the filtering problem of the two-dimensional partially observable process $\left(\left(X_{t}, Y_{t}\right), t \in[0, T]\right)$ governed by the following linear system of equations:

$$
\begin{gathered}
d X_{t}=\theta X_{t} d t+\sigma d V_{t}^{(1)}, \quad 0 \leq t \leq T, X_{0}=\rho \\
d Y_{t}=\mu X_{t} d t+d V_{t}^{(2)}, \quad 0 \leq t \leq T, Y_{0}=0
\end{gathered}
$$

Here $X=\left(X_{t}, t \in[0, T]\right)$ and $Y=\left(Y_{t}, t \in[0, T]\right)$ represent the state and the observation, respectively. For $j=1,2$, the noise process $V^{(j)}=\left(V_{t}^{(j)}, t \in[0, T]\right)$ is described by (1.1) with $(p, q)=\left(p_{j}, q_{j}\right)$ and $W_{t}=W_{t}^{(j)}, t \in \mathbb{R}$. We assume that the Brownian motions $\left(W_{t}^{(1)}, t \in \mathbb{R}\right)$ and $\left(W_{t}^{(2)}, t \in \mathbb{R}\right)$, whence $V^{(1)}$ and $V^{(2)}$, are independent. The coefficients $\theta, \sigma, \mu \in \mathbb{R}$ with $\mu \neq 0$ are known constants, and $\rho$ is a centered Gaussian random variable independent of $\left(V^{(1)}, V^{(2)}\right)$.

The basic filtering problem for the linear model (1.6) with memory is to calculate the conditional expectation $\mathbb{E}\left[X_{t} \mid \mathscr{F}_{t}^{Y}\right]$, called the (optimal) filter, where $\mathscr{F}^{Y}=\left(\mathscr{F}_{t}^{Y}, t \in\right.$ $[0, T])$ is the natural filtration of the observation process $Y$. In the classical Kalman-Bucy theory (see Kalman [12], Kalman and Bucy [13], Bucy and Joseph [4], Davis [5], and Liptser and Shiryaev [21]), Brownian motion is used as the driving noise. Attempts have been made to resolve the filtering problem of dynamical systems with memory by replacing Brownian motion by other processes. In [16-18] and others, fractional Brownian motion is used. Notice that fractional Brownian motion is not a semimartingale. 
In the discrete-time setting, autoregressive processes are used as driving noise (see, e.g., Kalman [12], Bucy and Joseph [4], and Jazwinski [11]). Our model may be regarded as a continuous-time analog of the latter since it is shown by Anh and Inoue [1] that $V$ is governed by a continuous-time $\operatorname{AR}(\infty)$-type equation (see Section 7 ).

The Kalman-Bucy filter is a computationally tractable scheme for the optimal filter of a Markovian system. We aim to derive a similar effective filtering algorithm for the system (1.6) which has memory. However, rather than considering (1.6) itself, we start with a general continuous Gaussian process $X=\left(X_{t}, t \in[0, T]\right)$ as the state process and $Y=\left(Y_{t}, t \in[0, T]\right)$ defined by

$$
Y_{t}=\int_{0}^{t} \mu(s) X_{s} d s+V_{t}, \quad 0 \leq t \leq T
$$

as the observation process, where $\mu(\cdot)$ is a deterministic function and $V=\left(V_{t}, t \in[0, T]\right)$ is a process which is independent of $X$ and given by (1.1). Using (1.4) and (1.5), we derive explicit Volterra integral equations for the optimal filter (Theorem 3.1). In the special case (1.6), the integral equations are reduced to differential equations, which give an extension to Kalman-Bucy filtering equations (Theorem 3.5). Due to the non-Markovianness of the formulation (1.6), it is expected that the resulting filtering equations would appear in the integral equation form (cf. Kleptsyna et al. [16]). The fact that good Kalman-Bucytype differential equations can be obtained here is due to the special properties of (1.6). This interesting result does not seem to hold for any other formulation where memory is inherent.

We apply the results to an optimal portfolio problem in a partially observable financial market model. More precisely, we consider a stock price model that is driven by the process $V=\left(V_{t}, t \in[0, T]\right)$ given by (1.1). Assuming that the investor can observe the stock price but not the drift process, we discuss the portfolio optimization problem of maximizing the expected logarithmic utility from terminal wealth. To solve this problem, we make use of our results on filtering to reduce the problem to the case where the drift process is adapted to the observation process. We then use the martingale methods (cf. Karatzas and Shreve [14]) to work out the explicit formula for the optimal portfolio (Theorem 4.1).

This paper is organized as follows. In Section 2, we prove the semimartingale representation (1.4) with (1.5) for $V$. Section 3 is the main body of this paper. We derive closed-form equations for the optimal filter. In Section 4 , we apply the results to finance. In Section 5, we illustrate the advantage of $V$ in parameter estimation. Some numerical results on Monte Carlo simulation are presented. In Section 6, we numerically compare the performance of our filter with the Kalman-Bucy filter in the presence of memory effect. Finally, a possible extension of this work is discussed in Section 7.

\section{Driving noise process with memory}

Let $T \in(0, \infty)$, and let $(\Omega, \mathscr{F}, \mathbb{P})$ be a complete probability space. For a process $\left(A_{t}, t \in\right.$ $[0, T])$, we denote by $\mathscr{F}^{A}=\left(\mathscr{F}_{t}^{A}, t \in[0, T]\right)$ the $\mathbb{P}$-augmentation of the filtration $\left(\sigma\left(A_{s}\right.\right.$, $s \in[0, t]), t \in[0, T])$. 
4 Linear filtering of systems with memory

Let $V=\left(V_{t}, t \in[0, T]\right)$ be the process given by (1.1). The process $V$ is a continuous Gaussian process with stationary increments. The aim of this section is to prove (1.4) with (1.5).

We define a process $\alpha=\left(\alpha_{t}, t \in[0, T]\right)$ by

$$
\alpha_{t}=\mathbb{E}\left[\int_{-\infty}^{t} p e^{-(q+p)(t-u)} d W_{u} \mid \mathscr{F}_{t} V\right], \quad 0 \leq t \leq T .
$$

Then there exists a one-dimensional Brownian motion $B=\left(B_{t}, t \in[0, T]\right)$, called the innovation process, satisfying $\mathscr{F}_{t}^{B}=\mathscr{F}_{t}^{V}, 0 \leq t \leq T$, and

$$
V_{t}=B_{t}-\int_{0}^{t} \alpha_{s} d s, \quad 0 \leq t \leq T
$$

(see, e.g., Liptser and Shiryaev [21, Theorem 7.16]). Thus, $V$ is an $\mathscr{F}^{V}$-semimartingale with representation (2.2).

To find a good representation of the process $\alpha$, we recall the following result from Anh et al. [2, Example 5.3]:

$$
\alpha_{t}=\int_{0}^{t} k(t, s) d V_{s}, \quad 0 \leq t \leq T
$$

with

$$
k(t, s)=p(2 q+p) \frac{(2 q+p) e^{q s}-p e^{-q s}}{(2 q+p)^{2} e^{q t}-p^{2} e^{-q t}}, \quad 0 \leq s \leq t \leq T .
$$

From the theory of Volterra integral equations, there exists a function $l(t, s) \in L^{2}\left([0, T]^{2}\right)$, called the resolvent of $k(t, s)$, such that, for almost every $0 \leq s \leq t \leq T$,

$$
\begin{aligned}
& l(t, s)-k(t, s)+\int_{s}^{t} l(t, u) k(u, s) d u=0 \\
& l(t, s)-k(t, s)+\int_{s}^{t} k(t, u) l(u, s) d u=0
\end{aligned}
$$

(see Davis [5, Chapter 4, Section 3] and Gripenberg et al. [9, Chapter 9]). Using $l(t, s)$, we have the following representation of $\alpha$ in terms of the innovation process $B$ :

$$
\alpha_{t}=\int_{0}^{t} l(t, s) d B_{s}, \quad 0 \leq t \leq T
$$

We will solve (2.5) explicitly to obtain $l(t, s)$.

Theorem 2.1. The expression (1.5) holds.

Proof. We have $k(t, s)=a(t) b(s)$ for $0 \leq s \leq t \leq T$, where, for $t \in[0, T]$,

$$
a(t)=\frac{p(2 q+p)}{(2 q+p)^{2} e^{q t}-p^{2} e^{-q t}}, \quad b(t)=(2 q+p) e^{q t}-p e^{-q t}
$$


A. Inoue et al. 5

Fix $s \in[0, T]$ and define $x(t)=x_{s}(t)$ and $\lambda=\lambda_{s}$ by

$$
x(t)=\int_{s}^{t} b(u) l(u, s) d u, \quad s \leq t \leq T, \lambda=b(s) .
$$

Then, from (2.5) we obtain

$$
\frac{d x}{d t}(t)+a(t) b(t) x(t)=\lambda a(t) b(t), \quad x(s)=0 .
$$

The solution $x$ is given by

$$
x(t)=\lambda-\lambda \exp \left[-\int_{s}^{t} a(u) b(u) d u\right]
$$

whence, for $0 \leq s \leq t \leq T$,

$$
l(t, s)=a(t) b(s) \exp \left[-\int_{s}^{t} a(u) b(u) d u\right]=k(t, s) \exp \left[-\int_{s}^{t} k(u, u) d u\right] .
$$

We have

$$
k(u, u)=p-\frac{2 p^{2} q}{(2 q+p)^{2} e^{2 q u}-p^{2}} .
$$

By the change of variable $x(u)=(2 q+p)^{2} e^{2 q u}-p^{2}$, we obtain

$$
\begin{aligned}
2 p^{2} q \int_{s}^{t} \frac{d u}{(2 q+p)^{2} e^{2 q u}-p^{2}} & =2 p^{2} q \int_{x(s)}^{x(t)} \frac{1}{2 q x\left(x+p^{2}\right)} d x \\
& =\int_{x(s)}^{x(t)}\left(\frac{1}{x}-\frac{1}{x+p^{2}}\right) d x=\log \left[\frac{x(t)\left(x(s)+p^{2}\right)}{x(s)\left(x(t)+p^{2}\right)}\right] \\
& =\log \left[e^{-2 q(t-s)} \frac{(2 q+p)^{2} e^{2 q t}-p^{2}}{(2 q+p)^{2} e^{2 q s}-p^{2}}\right],
\end{aligned}
$$

so that

$$
\exp \left[-\int_{s}^{t} k(u, u) d u\right]=e^{-p(t-s)} e^{-2 q(t-s)} \frac{(2 q+p)^{2} e^{2 q t}-p^{2}}{(2 q+p)^{2} e^{2 q s}-p^{2}}
$$

Thus

$$
l(t, s)=(2 q+p) p e^{-(p+q)(t-s)} \frac{(2 q+p) e^{2 q s}-p}{(2 q+p)^{2} e^{2 q s}-p^{2}}
$$

or (1.5) holds, as desired. 
6 Linear filtering of systems with memory

\section{Filtering equations}

3.1. General result. We consider a general two-dimensional centered continuous Gaussian process $\left(\left(X_{t}, U_{t}\right), t \in[0, T]\right)$. The process $X=\left(X_{t}, t \in[0, T]\right)$ represents the state process, while $U=\left(U_{t}, t \in[0, T]\right)$ is another process which is related to the dynamics of $X$.

In this subsection, $\left(B_{t}, t \in[0, T]\right)$ is not an innovation process but just a one-dimensional Brownian motion that is independent of $(X, U)$. Let $l(t, s)$ be an arbitrary Volterratype bounded measurable function on $[0, T]^{2}$ (i.e., $l(t, s)=0$ for $s>t$ ). Though the function given by (1.5) satisfies this assumption, (1.5) itself is not assumed in this subsection. We define the processes $\alpha=\left(\alpha_{t}, t \in[0, T]\right)$ and $V=\left(V_{t}, t \in[0, T]\right)$ by (2.6) and (2.2), respectively. Thus, in particular, $\alpha$ is not assumed to be given by a conditional expectation of the type (2.1), and $V$ is not necessarily a stationary increment process. We consider the observation process $Y=\left(Y_{t}, t \in[0, T]\right)$ satisfying

$$
Y_{t}=\int_{0}^{t} \mu(s) X_{s} d s+V_{t}, \quad 0 \leq t \leq T,
$$

where $\mu(\cdot)$ is a bounded measurable deterministic function on $[0, T]$ such that $\mu(t) \neq 0$ for $0 \leq t \leq T$.

Let $\mathscr{F}^{Y}=\left(\mathscr{F}_{t}^{Y}, t \in[0, T]\right)$ be the augmented filtration generated by $Y$. For $d$-dimensional column-vector processes $A=\left(A_{t}, t \in[0, T]\right)$ and $C=\left(C_{t}, t \in[0, T]\right)$, we write

$$
\begin{array}{cl}
\hat{A}_{t}=\mathbb{E}\left[A_{t} \mid \mathscr{F}_{t}^{Y}\right], & 0 \leq t \leq T, \\
\Gamma_{A C}(t, s)=\mathbb{E}\left[A_{t} C_{s}^{*}\right], & 0 \leq s \leq t \leq T,
\end{array}
$$

where $*$ denotes the transposition. Notice that $\Gamma_{A C}$ is $\mathbb{R}^{d \times d}$-valued.

Specifically, we consider

$$
Z_{t}=\left(X_{t}, U_{t}, \alpha_{t}\right)^{*}, \quad 0 \leq t \leq T,
$$

and define the error matrix $P(t, s) \in \mathbb{R}^{3 \times 3}$ by

$$
P(t, s)=\mathbb{E}\left[Z_{t}\left(Z_{s}-\hat{Z}_{s}\right)^{*}\right], \quad 0 \leq s \leq t \leq T \text {. }
$$

The next theorem gives an answer to the filtering problem for the partially observable process $\left(\left(X_{t}, Y_{t}\right), t \in[0, T]\right)$. It turns out that this will be useful in the filtering problem for (1.6) for example.

Theorem 3.1. The filter $\hat{Z}=\left(\hat{Z}_{t}, t \in[0, T]\right)$ satisfies the stochastic integral equation

$$
\hat{Z}_{t}=\int_{0}^{t}\{P(t, s)+D(t, s)\} a(s)\left\{d Y_{s}-a^{*}(s) \hat{Z}_{s} d s\right\}, \quad 0 \leq t \leq T,
$$


and the error matrix $P(t, s)$ is the unique solution to the following matrix Riccati-type integral equation such that $P(t, t)$ is symmetric and nonnegative definite for $0 \leq t \leq T$ :

$$
P(t, s)=-\int_{0}^{s}\{P(t, r)+D(t, r)\} a(r) a^{*}(r)\{P(s, r)+D(s, r)\}^{*} d r+\Gamma_{Z Z}(t, s), \quad 0 \leq s \leq t \leq T,
$$

where

$$
D(t, s)=\left(\begin{array}{ccc}
0 & 0 & 0 \\
0 & 0 & 0 \\
\frac{l(t, s)}{\mu(s)} & 0 & 0
\end{array}\right), \quad a(s)=\left(\begin{array}{c}
\mu(s) \\
0 \\
-1
\end{array}\right) \text {. }
$$

Proof. Since $(X, U)$ is independent of $B,(X, U, \alpha, Y)$ forms a Gaussian system. We have

$$
Y_{t}=\int_{0}^{t}\left(\mu(s) X_{s}-\alpha_{s}\right) d s+B_{t}
$$

Thus we can define the innovation process $I=\left(I_{t}, t \in[0, T]\right)$ by

$$
I_{t}=Y_{t}-\int_{0}^{t}\left(\mu(s) \hat{X}_{s}-\hat{\alpha}_{s}\right) d s, \quad 0 \leq t \leq T
$$

which is a Brownian motion satisfying $\mathscr{F}^{Y}=\mathscr{F}^{I}$ (cf. Liptser and Shiryaev [21, Theorem 7.16]). Notice that $I$ can be written as

$$
I_{t}=\int_{0}^{t}\left(Z_{s}-\hat{Z}_{s}\right)^{*} a(s) d s+B_{t}, \quad 0 \leq t \leq T
$$

By corollary to [21, Theorem 7.16], there exists an $\mathbb{R}^{3}$-valued Volterra-type function $F(t, s)=\left(F_{1}(t, s), F_{2}(t, s), F_{3}(t, s)\right)^{*}$ on $[0, T]^{2}$ such that

$$
\begin{gathered}
\int_{0}^{t}|F(t, s)|^{2} d s<+\infty, \quad 0 \leq t \leq T, \\
\hat{Z}_{t}=\int_{0}^{t} F(t, s) d I_{s}, \quad 0 \leq t \leq T,
\end{gathered}
$$

where $|\cdot|$ denotes the Euclidean norm.

Now let $g(t)=\left(g_{1}(t), g_{2}(t), g_{3}(t)\right)$ be an arbitrary bounded measurable row-vector function on $[0, T]$. Then, for $t \in[0, T]$,

$$
\mathbb{E}\left[\int_{0}^{t} g(s) d I_{s} \cdot\left(Z_{t}-\hat{Z}_{t}\right)\right]=0
$$


8 Linear filtering of systems with memory

From this, (3.10), (3.12), and the fact that $(X, U)$ and $B$ are independent, we have

$$
\begin{aligned}
\int_{0}^{t} g(s) F(t, s) d s & =\mathbb{E}\left[\int_{0}^{t} g(s) d I_{s} \cdot Z_{t}\right] \\
& =\mathbb{E}\left[\int_{0}^{t} g(s)\left\{\left(Z_{s}-\hat{Z}_{s}\right)^{*} a(s) d s+d B_{s}\right\} \cdot Z_{t}\right] \\
& =\int_{0}^{t} g(s) \mathbb{E}\left[Z_{t}\left(Z_{s}-\hat{Z}_{s}\right)^{*}\right] a(s) d s+\int_{0}^{t} g_{3}(s) l(t, s) d s \\
& =\int_{0}^{t} g(s) P(t, s) a(s) d s+\int_{0}^{t} g(s) D(t, s) a(s) d s .
\end{aligned}
$$

Since $g(\cdot)$ is arbitrary, we deduce that $F(t, s)=\{P(t, s)+D(t, s)\} a(s)$ or

$$
\hat{Z}_{t}=\int_{0}^{t}\{P(t, s)+D(t, s)\} a(s) d I_{s}, \quad 0 \leq t \leq T .
$$

The SDE (3.5) follows from (3.15) and the representation

$$
I_{t}=Y_{t}-\int_{0}^{t} a^{*}(s) \hat{Z}_{s} d s, \quad 0 \leq t \leq T .
$$

Equation (3.6) follows from (3.15) and the equality

$$
P(t, s)=\mathbb{E}\left[Z_{t} Z_{s}^{*}\right]-\mathbb{E}\left[\hat{Z}_{t} \hat{Z}_{s}^{*}\right]
$$

The matrix $P(t, t)$ is clearly symmetric and nonnegative definite. Finally, the uniqueness of the solution to (3.6) follows from Proposition 3.2.

Proposition 3.2. The solution $P(t, s)$ to the matrix integral equation (3.6) such that $P(t, t)$ is symmetric and nonnegative definite for $0 \leq t \leq T$ is unique.

Proof. By continuity, there exists a positive constant $C=C(T)$ such that

$$
\left\|\Gamma_{Z Z}(t, s)\right\| \leq C, \quad 0 \leq s \leq t \leq T,
$$

where $\|A\|:=\left\{\operatorname{trace}\left(A^{*} A\right)\right\}^{1 / 2}$ for $A \in \mathbb{R}^{3 \times 3}$. Let $P$ be a solution to (3.6) such that $P(t, t)$ is symmetric and nonnegative definite for $t \in[0, T]$. We put $Q(t, s)=P(t, s)+D(t, s)$. Then (3.6) with $s=t$ is

$$
\Gamma_{Z Z}(t, t)-P(t, t)=\int_{0}^{t} Q(t, r) a(r) a^{*}(r) Q^{*}(t, r) d r
$$

From this, we have

$$
\begin{aligned}
\int_{0}^{t}|Q(t, r) a(r)|^{2} d r & =\int_{0}^{t} \operatorname{trace}\left\{Q(t, r) a(r) a^{*}(r) Q^{*}(t, r)\right\} d r \\
& =\operatorname{trace}\left\{\Gamma_{Z Z}(t, t)-P(t, t)\right\} \leq \operatorname{trace}\left\{\Gamma_{Z Z}(t, t)\right\} \\
& \leq \sqrt{3}\left\|\Gamma_{Z Z}(t, t)\right\| \leq \sqrt{3} C .
\end{aligned}
$$


A. Inoue et al. 9

Therefore, $\|P(t, s)\|$ is at most

$$
\begin{aligned}
& \left\|\Gamma_{Z Z}(t, s)\right\|+\int_{0}^{s}\left\|Q(t, r) a(r) a^{*}(r) Q^{*}(s, r)\right\| d r \\
& \quad \leq\left\|\Gamma_{Z Z}(t, s)\right\|+\left\{\int_{0}^{t}|Q(t, r) a(r)|^{2} d r\right\}^{1 / 2} \cdot\left\{\int_{0}^{s}|Q(s, r) a(r)|^{2} d r\right\}^{1 / 2} \\
& \quad \leq(1+\sqrt{3}) C .
\end{aligned}
$$

Let $P_{1}$ and $P_{2}$ be two solutions of (3.6). We define $Q_{i}(t, s)=P_{i}(t, s)+D(t, s)$ for $i=1,2$. We put $P_{i}(t, s)=0$ for $s>t$ and $i=1,2$. Since $\mu$ and $l$ are bounded, it follows from the above estimate that there exists a positive constant $K=K(T)$ satisfying

$$
\left\|a(s) a^{*}(s) Q_{i}(t, s)\right\| \leq K, \quad 0 \leq s \leq t \leq T, i=1,2 .
$$

It follows that

$$
\begin{aligned}
&\left\|Q_{1}(t, r) a(r) a^{*}(r) Q_{1}(s, r)^{*}-Q_{2}(t, r) a(r) a^{*}(r) Q_{2}(s, r)^{*}\right\| \\
& \leq\left\|Q_{1}(t, r) a(r) a^{*}(r)\left\{Q_{1}(s, r)^{*}-Q_{2}(s, r)^{*}\right\}\right\| \\
&+\left\|\left\{Q_{1}(t, r)-Q_{2}(t, r)\right\} a(r) a^{*}(r) Q_{2}(s, r)^{*}\right\| \\
& \leq 2 K \sup _{0 \leq t \leq T}\left\|Q_{1}(t, r)-Q_{2}(t, r)\right\|=2 K \sup _{0 \leq t \leq T}\left\|P_{1}(t, r)-P_{2}(t, r)\right\| .
\end{aligned}
$$

From this and (3.6), we obtain

$$
\sup _{0 \leq t \leq T}\left\|P_{1}(t, s)-P_{2}(t, s)\right\| \leq 2 K \int_{0}^{s} \sup _{0 \leq t \leq T}\left\|P_{1}(t, r)-P_{2}(t, r)\right\| d r .
$$

Therefore, Gronwall's lemma gives

$$
\sup _{0 \leq t \leq T}\left\|P_{1}(t, s)-P_{2}(t, s)\right\|=0, \quad 0 \leq s \leq T .
$$

Thus the uniqueness follows.

Remark 3.3. We consider the case in which $\alpha=0$ and the state process $X$ is the OrnsteinUhlenbeck process satisfying

$$
d X_{t}=\theta X_{t} d t+\sigma d W_{t}, \quad 0 \leq t \leq T, X_{0}=0,
$$

where $\theta, \sigma \neq 0$ and $\left(W_{t}, t \in[0, T]\right)$ is a one-dimensional Brownian motion that is independent of $B$. We also assume that $\mu(\cdot)=\mu$, a constant. Then $X_{t}=\sigma \int_{0}^{t} e^{\theta(t-u)} d W_{u}$ and

$$
\Gamma_{X X}(t, s)=\frac{\sigma^{2}}{2 \theta}\left\{e^{\theta(t+s)}-e^{\theta(t-s)}\right\}, \quad 0 \leq s \leq t \leq T .
$$

By Theorem 3.1, we have

$$
\begin{gathered}
\hat{X}_{t}=\int_{0}^{t} \mu P_{X X}(t, s)\left\{d Y_{s}-\mu \hat{X}_{s} d s\right\}, \\
P_{X X}(t, s)=\Gamma_{X X}(t, s)-\int_{0}^{s} \mu^{2} P_{X X}(t, r) P_{X X}(s, r) d r,
\end{gathered}
$$


where $P_{X X}(t, s)=\mathbb{E}\left[X_{t}\left(X_{s}-\hat{X}_{s}\right)\right]$ for $0 \leq s \leq t \leq T$. Let $\left(\mathscr{F}_{t}, t \in[0, T]\right)$ be the $\mathbb{P}$-augmentation of the filtration generated by $\left(\left(W_{t}, B_{t}\right), t \in[0, T]\right)$. Then $P_{X X}(t, s)$ is

$$
\begin{aligned}
\mathbb{E}\left[X_{t}\left(X_{s}-\hat{X}_{s}\right)\right] & =\mathbb{E}\left[\mathbb{E}\left[X_{t} \mid \mathscr{F}(s)\right]\left(X_{s}-\hat{X}_{s}\right)\right] \\
& =\mathbb{E}\left[e^{\theta(t-s)} X_{s}\left(X_{s}-\hat{X}_{s}\right)\right]=e^{\theta(t-s)} \gamma(s)
\end{aligned}
$$

with $\gamma(s)=\mathbb{E}\left[X_{s}\left(X_{s}-\hat{X}_{s}\right)\right]$. Thus $(3.28)$ is reduced to

$$
d \hat{X}_{t}=\left\{\theta-\mu^{2} \gamma(t)\right\} \hat{X}_{t} d t+\mu \gamma(t) d Y_{t}
$$

Differentiating (3.29) in $s=t$, we get

$$
\frac{d \gamma(t)}{d t}=\sigma^{2}+2 \theta \gamma(t)-\mu^{2} \gamma(t)^{2}
$$

Equations (3.31) and (3.32) are the well-known Kalman-Bucy filtering equations (see Kalman and Bucy [13], Bucy and Joseph [4], Davis [5], Jazwinski [11], and Liptser and Shiryaev [21]).

3.2. Linear systems with memory. We turn to the partially observable system governed by (1.6).

Let $\left(W_{t}^{(j)}, t \in \mathbb{R}\right), j=1,2$, be two independent Brownian motions. For $j=1,2$, let $V^{(j)}=\left(V_{t}^{(j)}, t \in[0, T]\right)$ be the noise process described by (1.1) with $W_{t}=W_{t}^{(j)}, t \in \mathbb{R}$, and $(p, q)=\left(p_{j}, q_{j}\right)$ satisfying (1.2). Then $V^{(1)}$ and $V^{(2)}$ are independent. Let $\left(\left(X_{t}, Y_{t}\right), t \in\right.$ $[0, T])$ be the two-dimensional process satisfying (1.6), where the coefficients $\theta, \sigma, \mu \in \mathbb{R}$ with $\mu \neq 0$ are known constants and the initial value $\rho$ is a centered Gaussian random variable that is independent of $\left(V^{(1)}, V^{(2)}\right)$. The processes $X$ and $Y$ represent the state and the observation, respectively.

By Theorem 2.1, we have, for $j=1,2$ and $t \in[0, T]$,

$$
\alpha_{t}^{(j)}=\int_{0}^{t} l_{j}(t, s) d B_{s}^{(j)}, \quad V_{t}^{(j)}=B_{t}^{(j)}-\int_{0}^{t} \alpha_{s}^{(j)} d s
$$

where $B^{(j)}=\left(B_{t}^{(j)}, t \in[0, T]\right), j=1,2$, are two independent Brownian motions and

$$
l_{j}(t, s)=p_{j} e^{-\left(p_{j}+q_{j}\right)(t-s)}\left\{1-\frac{2 p_{j} q_{j}}{\left(2 q_{j}+p_{j}\right)^{2} e^{2 q_{j} s}-p_{j}^{2}}\right\}, \quad 0 \leq s \leq t \leq T .
$$

We put $l_{j}(t)=l_{j}(t, t)$ for $j=1,2$, that is,

$$
l_{j}(t)=p_{j}\left\{1-\frac{2 p_{j} q_{j}}{\left(2 q_{j}+p_{j}\right)^{2} e^{2 q_{j} s}-p_{j}^{2}}\right\}, \quad 0 \leq t \leq T .
$$

It holds that $l_{j}(t, s)=e^{-r_{j}(t-s)} l_{j}(s)$ with

$$
r_{j}=p_{j}+q_{j}, \quad j=1,2 .
$$


A. Inoue et al. 11

We denote by $\mathscr{F}_{\mathrm{F}}=\left(\mathscr{F}_{t}, t \in[0, T]\right)$ the $\mathbb{P}$-augmentation of the filtration $\left(\sigma\left(\rho,\left(V_{s}^{(1)}\right.\right.\right.$, $\left.\left.\left.V_{s}^{(2)}\right)_{s \in[0, t]}\right), t \in[0, T]\right)$ generated by the initial value $X_{0}=\rho$ and the noise process $\left(V^{(1)}\right.$, $\left.V^{(2)}\right)$.

LEMma 3.4. It holds that

$$
\mathbb{E}\left[X_{t} \mid \mathscr{F}_{s}\right]=e^{\theta(t-s)} X_{s}-\sigma b(t-s) \alpha_{s}^{(1)}, \quad 0 \leq s \leq t \leq T,
$$

where

$$
b(t)= \begin{cases}\frac{e^{\theta t}-e^{-r_{1} t}}{\theta+r_{1}}, & \theta+r_{1} \neq 0, \\ t e^{\theta t}, & \theta+r_{1}=0 .\end{cases}
$$

Proof. For $t \in[0, T], \int_{0}^{t} e^{\theta(t-s)} \alpha_{s}^{(1)} d s$ is

$$
\int_{0}^{t} l_{1}(u)\left\{\int_{u}^{t} e^{\theta(t-s)} e^{-r_{1}(s-u)} d s\right\} d B_{u}^{(1)}=\int_{0}^{t} b(t-u) l_{1}(u) d B_{u}^{(1)} .
$$

Since $X_{t}=e^{\theta t} X_{0}+\sigma \int_{0}^{t} e^{\theta(t-u)} d V_{u}^{(1)}$ or

$$
X_{t}=e^{\theta t} X_{0}+\sigma \int_{0}^{t} e^{\theta(t-u)} d B_{u}^{(1)}-\sigma \int_{0}^{t} e^{\theta(t-u)} \alpha_{u}^{(1)} d u,
$$

$\mathbb{E}\left[X_{t} \mid \mathscr{F}_{s}\right]$ with $s \leq t$ is equal to

$$
\begin{aligned}
e^{\theta t} X_{0} & +\sigma e^{\theta(t-s)} \int_{0}^{s} e^{\theta(s-u)} d B_{u}^{(1)}-\sigma \int_{0}^{s} b(t-u) l_{1}(u) d B_{u}^{(1)} \\
& =e^{\theta(t-s)} X_{s}-\sigma \int_{0}^{s}\left\{b(t-u)-e^{\theta(t-s)} b(s-u)\right\} l_{1}(u) d B_{u}^{(1)} .
\end{aligned}
$$

However, by elementary calculation, we have

$$
b(t-u)-e^{\theta(t-s)} b(s-u)=b(t-s) e^{-r_{1}(s-u)}, \quad 0 \leq u \leq s \leq t .
$$

Thus the lemma follows.

We put, for $0 \leq t \leq T$,

$$
\begin{array}{ccc}
F=\left(\begin{array}{ccc}
-\theta & \sigma & 0 \\
0 & r_{1} & 0 \\
0 & 0 & r_{2}
\end{array}\right), \quad D(t)=\left(\begin{array}{ccc}
0 & 0 & 0 \\
0 & 0 & 0 \\
\mu^{-1} l_{2}(t) & 0 & 0
\end{array}\right), \quad a=\left(\begin{array}{c}
\mu \\
0 \\
-1
\end{array}\right), \\
G(t)=\left(\begin{array}{ccc}
\sigma^{2} & \sigma l_{1}(t) & 0 \\
\sigma l_{1}(t) & l_{1}^{2}(t) & 0 \\
0 & 0 & 0
\end{array}\right), & H(t)=\left(\begin{array}{ccc}
-\theta & \sigma & 0 \\
0 & r_{1} & 0 \\
\mu l_{2}(t) & 0 & r_{2}-l_{2}(t)
\end{array}\right) .
\end{array}
$$

We also put

$$
Z_{t}=\left(X_{t}, \alpha_{t}^{(1)}, \alpha_{t}^{(2)}\right)^{*}, \quad 0 \leq t \leq T
$$


12 Linear filtering of systems with memory

Recall that $\Gamma_{Z Z}(0)=\mathbb{E}\left[Z_{0} Z_{0}^{*}\right]$ and that

$$
\hat{Z}_{t}=\mathbb{E}\left[Z_{t} \mid \mathscr{F}_{t}^{Y}\right], \quad 0 \leq t \leq T
$$

We define the error matrix $P(t) \in \mathbb{R}^{3 \times 3}$ by

$$
P(t)=\mathbb{E}\left[Z_{t}\left(Z_{t}-\hat{Z}_{t}\right)^{*}\right], \quad 0 \leq t \leq T .
$$

Here is the solution to the optimal filtering problem for (1.6).

TheOREM 3.5. The filter $\hat{Z}=\left(\hat{Z}_{t}, t \in[0, T]\right)$ satisfies the stochastic differential equation

$$
d \hat{Z}_{t}=-\left\{F+(P(t)+D(t)) a a^{*}\right\} \hat{Z}_{t} d t+(P(t)+D(t)) a d Y_{t}, \quad 0 \leq t \leq T,
$$

with $\hat{Z}_{0}=(0,0,0)^{*}$, and $P(\cdot)$ follows the matrix Riccati equation

$$
\frac{d P(t)}{d t}=G(t)-H(t) P(t)-P(t) H(t)^{*}-P(t) a a^{*} P(t), \quad 0 \leq t \leq T,
$$

with $P_{i j}(0)=\delta_{i 1} \delta_{j 1} \mathbb{E}\left[\rho^{2}\right]$ for $i, j=1,2,3$.

Proof. For $0 \leq s \leq t \leq T$, we put

$$
P(t, s)=\mathbb{E}\left[Z_{t}\left(Z_{s}-\hat{Z}_{s}\right)^{*}\right]
$$

Then we have $P(t)=P(t, t)$. We also put, for $0 \leq s \leq t \leq T$,

$$
\begin{gathered}
D(t, s)=e^{-r_{2}(t-s)} D(s), \\
Q(t, s)=P(t, s)+D(t, s), \quad Q(s)=Q(s, s)=P(s)+D(s) .
\end{gathered}
$$

By

$$
P(t, s)=\mathbb{E}\left[\mathbb{E}\left[Z_{t} \mid \mathscr{F}(s)\right] \cdot\left(Z_{s}-\hat{Z}_{s}\right)^{*}\right]
$$

and Lemma 3.4, we have $P(t, s)=M(t-s) P(s)$, where

$$
M(t)=\left(\begin{array}{ccc}
e^{\theta t} & -\sigma b(t) & 0 \\
0 & e^{-r_{1} t} & 0 \\
0 & 0 & e^{-r_{2} t}
\end{array}\right)
$$

with $b(\cdot)$ as in Lemma 3.4. We also see that $D(t, s)=M(t-s) D(s)$. Combining, $Q(t, s)=$ $M(t-s) Q(s)$. However, $M(t)=e^{-t F}$ since $d M(t) / d t=-F M(t)$ and $M(0)$ is the unit matrix. Thus we obtain

$$
Q(t, s)=e^{-(t-s) F} Q(s) .
$$


From (3.53) and Theorem 3.1 with $U=\alpha^{(1)}$ and $\alpha=\alpha^{(2)}$, it follows that

$$
\begin{gathered}
\hat{Z}_{t}=\int_{0}^{t} e^{-(t-s) F} Q(s) a\left\{d Y_{s}-a^{*} \hat{Z}_{s} d s\right\}, \\
P(t)=\Gamma_{Z Z}(t)-\int_{0}^{t} e^{-(t-u) F} Q(u) a a^{*} Q^{*}(u) e^{-(t-u) F^{*}} d u .
\end{gathered}
$$

The SDE (3.47) follows easily from (3.54).

Differentiating both sides of (3.55), we get

$$
\begin{aligned}
\dot{P} & =\dot{\Gamma}_{Z Z}+F\left(\Gamma_{Z Z}-P\right)+\left(\Gamma_{Z Z}-P\right) F^{*}-Q a a^{*} Q^{*} \\
& =\dot{\Gamma}_{Z Z}+F \Gamma_{Z Z}+\Gamma_{Z Z} F^{*}-D a a^{*} D-H P-P H^{*}-P a a^{*} P .
\end{aligned}
$$

Since $d Z_{t}=-F Z_{t} d t+d R_{t}$ with

$$
R_{t}=\left(\sigma B_{t}^{(1)}, \int_{0}^{t} l_{1}(s) d B_{s}^{(1)}, \int_{0}^{t} l_{2}(s) d B_{s}^{(2)}\right)^{*}
$$

we see by integration by parts that $Z_{t} Z_{t}^{*}-Z_{0} Z_{0}^{*}$ is equal to

$$
\int_{0}^{t} Z_{s} d Z_{s}^{*}+\int_{0}^{t} d Z_{s} Z_{s}^{*}+\mathbb{E}\left[R_{t} R_{t}^{*}\right]
$$

It follows that $\Gamma_{Z Z}(t)-\Gamma_{Z Z}(0)$ is equal to

$$
\mathbb{E}\left[\int_{0}^{t} Z_{s} d Z_{s}^{*}\right]+\mathbb{E}\left[\int_{0}^{t} d Z_{s} Z_{s}^{*}\right]+\mathbb{E}\left[R_{t} R_{t}^{*}\right]=-\int_{0}^{t} \Gamma_{Z Z}(s) F^{*} d s-\int_{0}^{t} F \Gamma_{Z Z}(s) d s+\mathbb{E}\left[R_{t} R_{t}^{*}\right]
$$

Thus

$$
\begin{aligned}
\Gamma_{Z Z}(t) & +\int_{0}^{t} F \Gamma_{Z Z}(s) d s+\int_{0}^{t} \Gamma_{Z Z}(s) F^{*} d s-\Gamma_{Z Z}(0) \\
= & \mathbb{E}\left[R_{t} R_{t}^{*}\right]=\int_{0}^{t}\left\{G(s)+D(s) a a^{*} D^{*}(s)\right\} d s .
\end{aligned}
$$

This and (3.56) yield (3.48).

Remark 3.6. Suppose that, as in Section 3.1, the processes $\alpha^{(j)}$ and $V^{(j)}, j=1,2$, are defined by (3.33) with arbitrary Volterra-type bounded measurable functions $l_{j}(t, s)$ and Brownian motions $B^{(j)}$. If we further assume that $l_{j}(t, s), j=1,2$, are of the form $l_{j}(t, s)=$ $e^{c_{j}(t-s)} g_{j}(s)$, then we can easily extend Theorem 3.5 to this setting. Notice that, in this case, the noise processes $V^{(1)}$ and $V^{(2)}$ are not necessarily stationary increment processes.

Corollary 3.7. Assume that $p_{2}=0$, that is, $V_{t}^{(2)}=W_{t}^{(2)}, 0 \leq t \leq T$. For $t \in[0, T]$, let $Z_{t}=\left(X_{t}, \alpha_{t}^{(1)}\right)^{*}$ and $P(t)=\mathbb{E}\left[Z_{t}\left(Z_{t}-\hat{Z}_{t}\right)^{*}\right] \in \mathbb{R}^{2 \times 2}$. Then the filter $\hat{Z}=\left(\hat{Z}_{t}, t \in[0, T]\right)$ 
14 Linear filtering of systems with memory

and the error matrix $P(\cdot)$ satisfy, respectively,

$$
\begin{gathered}
d \hat{Z}_{t}=-\left\{F+P(t) a a^{*}\right\} \hat{Z}_{t} d t+P(t) a d Y_{t}, \quad 0 \leq t \leq T, \\
\frac{d P(t)}{d t}=G(t)-F P(t)-P(t) F^{*}-P(t) a a^{*} P(t), \quad 0 \leq t \leq T,
\end{gathered}
$$

with $\hat{Z}_{0}=(0,0)^{*}$ and $P_{i j}(0)=\delta_{i 1} \delta_{j 1} \mathbb{E}\left[\rho^{2}\right]$, where

$$
F=\left(\begin{array}{cc}
-\theta & \sigma \\
0 & r_{1}
\end{array}\right), \quad a=\left(\begin{array}{c}
\mu \\
0
\end{array}\right), \quad G(t)=\left(\begin{array}{cc}
\sigma^{2} & \sigma l_{1}(t) \\
\sigma l_{1}(t) & l_{1}(t)^{2}
\end{array}\right) .
$$

Corollary 3.8. Assume that $p_{1}=0$, that is, $V_{t}^{(1)}=W_{t}^{(1)}, 0 \leq t \leq T$. For $t \in[0, T]$, let $Z_{t}=\left(X_{t}, \alpha_{t}^{(2)}\right)^{*}$ and $P(t)=\mathbb{E}\left[Z_{t}\left(Z_{t}-\hat{Z}_{t}\right)^{*}\right] \in \mathbb{R}^{2 \times 2}$. Then the filter $\hat{Z}=\left(\hat{Z}_{t}, t \in[0, T]\right)$ and the error matrix $P(\cdot)$ satisfy, respectively,

$$
\begin{gathered}
d \hat{Z}_{t}=-\left\{F+(P(t)+D(t)) a a^{*}\right\} \hat{Z}_{t} d t+(P(t)+D(t)) a d Y_{t}, \quad 0 \leq t \leq T, \\
\frac{d P(t)}{d t}=G-H(t) P(t)-P(t) H(t)^{*}-P(t) a a^{*} P(t), \quad 0 \leq t \leq T,
\end{gathered}
$$

with $\hat{Z}_{0}=(0,0) *$ and $P_{i j}(0)=\delta_{i 1} \delta_{j 1} \mathbb{E}\left[\rho^{2}\right]$, where

$$
\begin{gathered}
F=\left(\begin{array}{cc}
-\theta & 0 \\
0 & r_{2}
\end{array}\right), \quad D(t)=\left(\begin{array}{cc}
0 & 0 \\
\mu^{-1} l_{2}(t) & 0
\end{array}\right), \quad a=\left(\begin{array}{c}
\mu \\
-1
\end{array}\right), \\
G=\left(\begin{array}{cc}
\sigma^{2} & 0 \\
0 & 0
\end{array}\right), \quad H(t)=\left(\begin{array}{cc}
-\theta & 0 \\
\mu l_{2}(t) & r_{2}-l_{2}(t)
\end{array}\right) .
\end{gathered}
$$

Example 3.9. We consider the estimation problem of the value of a signal $\rho$ from the observation process $Y=\left(Y_{t}, t \in[0, T]\right)$ given by

$$
d Y_{t}=\rho d t+d V_{t}, \quad 0 \leq t \leq T, Y_{0}=0,
$$

where $V=\left(V_{t}, t \in[0, T]\right)$ and $\alpha=\left(\alpha_{t}, t \in[0, T]\right)$ are as in Section 2. We assume that $\rho$ is a Gaussian random variable with mean zero and variance $v$. This is the special case $\theta=$ $\sigma=0, \mu=1$ of the setting of Corollary 3.8. Let $r=p+q$ and $l(\cdot)$ be as above. Let $H(t)$ and $a$ be as in Corollary 3.8 with $\mu=1$ and $\theta=0$. We define $P(t)=\left(P_{i j}(t)\right)_{1 \leq i, j \leq 2} \in \mathbb{R}^{2 \times 2}$ by $P(t)=\mathbb{E}\left[Z_{t}^{*}\left(Z_{t}-\hat{Z}_{t}\right)\right]$ with $Z_{t}=\left(\rho, \alpha_{t}\right)^{*}$. Then, by Corollary 3.8 , the filter $\hat{Z}_{t}=\left(\hat{\rho}_{t}, \hat{\alpha}_{t}\right)^{*}$ satisfies, for $t \in[0, T]$,

$$
\begin{aligned}
d \hat{\rho}(t)= & -\left(P_{11}(t)-P_{12}(t)\right)\left(\hat{\rho}_{t}-\hat{\alpha}_{t}\right) d t+\left(P_{11}(t)-P_{12}(t)\right) d Y_{t}, \\
d \hat{\alpha}_{t}= & \left\{\left(P_{21}(t)-P_{22}(t)+l(t)-r\right) \hat{\alpha}_{t}-\left(P_{21}(t)-P_{22}(t)+l(t)\right) \hat{\rho}_{t}\right\} d t \\
& +\left(P_{21}(t)-P_{22}(t)+l(t)\right) d Y_{t}
\end{aligned}
$$

with $\left(\hat{\rho}_{0}, \hat{\alpha}_{0}\right)=(0,0)$, and the error matrix $P(\cdot)$ follows

$$
\frac{d P(t)}{d t}=-H(t) P(t)-P(t) H(t)^{*}-P(t) a a^{*} P(t), \quad 0 \leq t \leq T,
$$


with $P_{i j}(0)=\delta_{i 1} \delta_{j 1} v$. By the linearization method as described in Bucy and Joseph [4, Chapter 5], we can solve the equation for $P(\cdot)$ to obtain

$$
P(t)=\frac{v}{1+v \eta(t)+v \xi(t) \phi(t)}\left(\begin{array}{cr}
1 & -\frac{\phi(t)}{\psi(t)} \\
-\frac{\phi(t)}{\psi(t)} & \frac{v \phi(t)^{2}}{\psi(t)^{2}}
\end{array}\right),
$$

where

$$
\begin{gathered}
\psi(t)=\exp \left\{\int_{0}^{t}(r-l(s)) d s\right\}, \quad \phi(t)=\int_{0}^{t} l(s) \psi(s) d s, \\
\xi(t)=\int_{0}^{t} \frac{\psi(s)+\phi(s)}{\psi(s)^{2}} d s, \quad \eta(t)=\int_{0}^{t}\left\{1-l(s) \psi(s) \xi(s)+\frac{\phi(s)}{\psi(s)}\right\} d s .
\end{gathered}
$$

The analytical forms of $\psi(\cdot), \phi(\cdot), \xi(\cdot)$, and $\eta(\cdot)$ can be derived. We omit the details.

\section{Application to finance}

In this section, we apply the results in the previous section to the problem of expected utility maximization for an investor with partial observations.

Let the processes $V^{(j)}=\left(V^{(j)}(t), t \in[0, T]\right), \alpha^{(j)}=\left(\alpha_{t}^{(j)}, t \in[0, T]\right), j=1,2$, be as in Section 3. In particular, $V^{(1)}$ and $V^{(2)}$ are independent. We consider the financial market consisting of a share of the money market with price $S_{t}^{0}$ at time $t \in[0, T]$ and a stock with price $S_{t}$ at time $t \in[0, T]$. The stock price process $S=\left(S_{t}, t \in[0, T]\right)$ is governed by the stochastic differential equation

$$
d S_{t}=S_{t}\left(U_{t} d t+\eta d V_{t}^{(2)}\right), \quad 0 \leq t \leq T, S_{0}=s_{0}
$$

where $s_{0}$ and $\eta$ are positive constants and $U=\left(U_{t}, t \in[0, T]\right)$ is a Gaussian process following

$$
d U_{t}=\left(\delta+\theta U_{t}\right) d t+\sigma d V_{t}^{(1)}, \quad 0 \leq t \leq T, U_{0}=\rho .
$$

The parameters $\theta, \delta$, and $\sigma$ are real constants, and $\rho$ is a Gaussian random variable that is independent of $\left(V^{(1)}, V^{(2)}\right)$. For simplicity, we assume that

$$
S_{t}^{0}=1, \quad 0 \leq t \leq T, \quad \eta=1, \quad \delta=0, \quad \mathbb{E}[\rho]=0 .
$$

Let $\mathscr{F}=\left(\mathscr{F}_{t}, t \in[0, T]\right)$ be the $\mathbb{P}$-augmentation of the filtration generated by the process $\left(\left(V_{t}^{(1)}, V_{t}^{(2)}\right), t \in[0, T]\right)$ and the random variable $\rho$. Then $U$ is $\mathscr{F}$-adapted but not $\mathscr{F}^{S}$-adapted; recall from Section 2 that $\mathscr{F}^{S}$ is the augmented filtration generated by the process $S$. Suppose that we can observe neither the disturbance process $V^{(2)}$ nor the drift process $U$ but only the price process $S$. Thus only $\mathscr{F}^{S}$-adapted processes are observable.

In many references such as Detemple [6], Dothan and Feldman [7], and Gennotte [8], the partially observable model described by (4.1) and (4.2) with $V^{(j)}$ 's replaced by Brownian motions, that is, $V^{(j)}=B^{(j)}$, is studied. 
We consider the following expected logarithmic utility maximization from terminal wealth: for given initial capital $x \in(0, \infty)$,

$$
\text { maximize } \mathbb{E}\left[\log \left(X_{T}^{x, \pi}\right)\right] \quad \text { over all } \pi \in \mathscr{A}(x) \text {, }
$$

where

$$
\begin{gathered}
\mathscr{A}(x)=\left\{\begin{array}{c}
\pi=\left(\pi_{t}, t \in[0, T]\right) \text { is } \mathbb{R} \text {-valued, } \mathscr{F}^{S} \text {-progressively } \\
\pi: \quad \text { measurable, } \int_{0}^{T} \pi_{t}^{2} d t<\infty, X_{t}^{x, \pi} \geq 0, t \in[0, T], \text { a.s. }
\end{array}\right\}, \\
X_{t}^{x, \pi}=x+\int_{0}^{t} \frac{\pi_{u}}{S_{u}} d S_{u}, \quad 0 \leq t \leq T .
\end{gathered}
$$

The value $\pi_{t}$ is the dollar amount invested in the stock at time $t$, whence $\pi_{t} / S_{t}$ is the number of units of stock held at time $t$. The process $X^{x, \pi}=\left(X_{t}^{x, \pi}, t \in[0, T]\right)$ is the wealth process associated with the self-financing portfolio determined uniquely by $\pi$.

An analog of the problem (4.4) for full observations is solved by Anh et al. [2]. For related work, see Karatzas and Zhao [15], Lakner [19, 20], and the references therein. We solve the problem (4.4) by combining the results above on filtering and the martingale method as described by Karatzas and Shreve [14].

Solving (4.1), we obtain

$$
S_{t}=s_{0} \exp \left(Y_{t}-\frac{1}{2} t\right), \quad 0 \leq t \leq T
$$

where the process $Y=\left(Y_{t}, t \in[0, T]\right)$ is given by

$$
Y_{t}=\int_{0}^{t} U_{s} d s+V_{t}^{(2)}=B_{t}^{(2)}+\int_{0}^{t}\left(U_{s}-\alpha_{s}^{(2)}\right) d s, \quad 0 \leq t \leq T
$$

From (4.7), we see that $\mathscr{F}^{S}=\mathscr{F} Y$. We regard $Y$ as the observation process. As in the previous sections, for a $d$-dimensional column-vector process $A=\left(A_{t}, t \in[0, T]\right)$, we write $\hat{A}_{t}=\mathbb{E}\left[A_{t} \mid \mathscr{F}_{t}^{Y}\right]$. In particular, $\hat{\alpha}_{t}^{(2)}=\mathbb{E}\left[\alpha_{t}^{(2)} \mid \mathscr{F}_{t}^{Y}\right]$. Let $I=\left(I_{t}, t \in[0, T]\right)$ be the innovation process associated with $Y$ that is given by

$$
I_{t}=Y_{t}-\int_{0}^{t}\left(\hat{U}_{s}-\hat{\alpha}_{s}^{(2)}\right) d s, \quad 0 \leq t \leq T
$$

The innovation process $I$ is an $\mathscr{F}^{Y}$-Brownian motion satisfying $\mathscr{F}^{S}=\mathscr{F}^{Y}=\mathscr{F}^{I}$ (see, e.g., Liptser and Shiryaev [21, Theorem 7.16]).

Let $L=\left(L_{t}, t \in[0, T]\right)$ be the exponential $\mathscr{F}$-martingale given by

$$
\begin{aligned}
L_{t} & =\exp \left\{-\int_{0}^{t}\left(U_{s}-\alpha_{s}^{(2)}\right) d B_{s}^{(2)}-\frac{1}{2} \int_{0}^{t}\left(U_{s}-\alpha_{s}^{(2)}\right)^{2} d s\right\} \\
& =\exp \left\{-\int_{0}^{t}\left(U_{s}-\alpha_{s}^{(2)}\right) d Y_{s}+\frac{1}{2} \int_{0}^{t}\left(U_{s}-\alpha_{s}^{(2)}\right)^{2} d s\right\} .
\end{aligned}
$$


We find that, for $t \in[0, T]$,

$$
\begin{aligned}
\hat{L}_{t} & =\exp \left\{-\int_{0}^{t}\left(\hat{U}_{s}-\hat{\alpha}_{s}^{(2)}\right) d Y_{s}+\frac{1}{2} \int_{0}^{t}\left(\hat{U}_{s}-\hat{\alpha}_{s}^{(2)}\right)^{2} d s\right\} \\
& =\exp \left\{-\int_{0}^{t}\left(\hat{U}_{s}-\hat{\alpha}_{s}^{(2)}\right) d I_{s}-\frac{1}{2} \int_{0}^{t}\left(\hat{U}_{s}-\hat{\alpha}_{s}^{(2)}\right)^{2} d s\right\}
\end{aligned}
$$

(see, e.g., Liptser and Shiryaev [21, Chapter 7]). The process $\widehat{L}=\left(\widehat{L}_{t}, t \in[0, T]\right)$ is an $\mathscr{F}^{Y}$-martingale.

For $x \in(0, \infty)$ and $\pi \in \mathscr{A}(x)$, we see from the Itô formula that the process $\left(\hat{L}_{t} X_{t}^{x, \pi}, t \in\right.$ $[0, T])$ is a local $\mathscr{F}^{Y}$-martingale, whence an $\mathscr{F}^{Y}$-supermartingale since $X^{x, \pi}$ is nonnegative. It follows that, for $x \in(0, \infty), \pi \in \mathscr{A}(x)$, and $y \in(0, \infty)$,

$$
\begin{aligned}
\mathbb{E}\left[\log \left(X_{T}^{x, \pi}\right)\right] & \leq \mathbb{E}\left[\log \left(X_{T}^{x, \pi}\right)-y \hat{L}_{T} X_{T}^{x, \pi}\right]+y x \\
& \leq \mathbb{E}\left[\log \left\{\frac{1}{\left(y \hat{L}_{T}\right)}\right\}-1\right]+y x,
\end{aligned}
$$

where, in the second inequality, we have used

$$
\log (z)-y z \leq \log \frac{1}{y}-1, \quad y, z \in(0, \infty) .
$$

The equalities in (4.12) hold if and only if

$$
X_{T}^{x, \pi}=\frac{x}{\widehat{L}_{T}} \quad \text { a.s. }
$$

Thus the portfolio process $\pi$ satisfying (4.14) is optimal.

Put

$$
\pi_{t}^{0}=x \frac{\left(\hat{U}_{t}-\hat{\alpha}_{t}^{(2)}\right)}{\hat{L}_{t}}, \quad 0 \leq t \leq T .
$$

Since $x / \widehat{L}_{0}=x$ and

$$
d \frac{x}{\hat{L}_{t}}=\frac{x\left(\hat{U}_{t}-\hat{\alpha}_{t}^{(2)}\right)}{\hat{L}_{t}} d Y_{t}=\frac{\pi_{t}^{0}}{S_{t}} d S_{t},
$$

we see from (4.6) that the process $\pi^{0}$ satisfies (4.14), whence it is the desired optimal portfolio process. It also satisfies

$$
\frac{\pi_{t}^{0}}{X_{t}^{x, \pi^{0}}}=\hat{U}_{t}-\hat{\alpha}_{t}^{(2)}, \quad 0 \leq t \leq T .
$$

We put

$$
a=(1,0,-1)^{*}, \quad Z_{t}=\left(U_{t}, \alpha_{t}^{(1)}, \alpha_{t}^{(2)}\right)^{*}, \quad 0 \leq t \leq T .
$$


We define the error matrix $P(t) \in \mathbb{R}^{3 \times 3}$ by $\mathbb{E}\left[Z_{t}\left(Z_{t}-\hat{Z}_{t}\right)^{*}\right]$. Combining the results above with Theorem 3.5 which describes the dynamics of $\hat{U}$ and $\hat{\alpha}^{(2)}$, we obtain the next theorem.

Theorem 4.1. The optimal portfolio process $\pi^{0}=\left(\pi_{t}^{0}, t \in[0, T]\right)$ for the problem (4.4) is given by

$$
\pi_{t}^{0}=\frac{x a^{*} \hat{Z}_{t}}{\hat{L}_{t}}, \quad 0 \leq t \leq T
$$

and satisfies

$$
X_{T}^{x, \pi^{0}}=\frac{x}{\hat{L}_{T}}, \quad \frac{\pi_{t}^{0}}{X_{t}^{x, \pi^{0}}}=a^{*} \hat{Z}_{t}, \quad 0 \leq t \leq T .
$$

The filter $\hat{Z}=\left(\hat{Z}_{t}, t \in[0, T]\right)$ follows

$$
d \hat{Z}_{t}=-\left\{F+(P(t)+D(t)) a a^{*}\right\} \hat{Z}_{t} d t+(P(t)+D(t)) a d Y_{t}, \quad 0 \leq t \leq T,
$$

with $\hat{Z}_{0}=(0,0,0)^{*}$, and the error matrix $P(\cdot)$ satisfies the matrix Riccati equation

$$
\frac{d P(t)}{d t}=G(t)-H(t) P(t)-P(t) H(t)^{*}-P(t) a a^{*} P(t), \quad 0 \leq t \leq T,
$$

with $P_{i j}(0)=\delta_{i 1} \delta_{j 1} \mathbb{E}\left[\rho^{2}\right](i, j=1,2,3)$, where $F, D(t), G(t)$, and $H(t)$ are as in Theorem 3.5 with $\mu=1$.

\section{Parameter estimation}

Let $V=\left(V_{t}, t \in[0, T]\right)$ be the process given by (1.1). We can estimate the values of the parameters $p$ and $q$ there from given data of $V$ by a least-squares approach (cf. Anh et al. [3]). In fact, since $V$ is a stationary increment process, the variance of $V_{t}-V_{s}$ is a function in $t-s$. To be precise,

$$
\frac{1}{t-s} \operatorname{Var}\left(V_{t}-V_{s}\right)=U(t-s), \quad 0 \leq s<t
$$

where the function $U(t)=U(t ; p, q)$ is given by

$$
U(t)=\frac{q^{2}}{(p+q)^{2}}+\frac{p(2 q+p)}{(p+q)^{3}} \cdot \frac{\left(1-e^{-(p+q) t}\right)}{t}, \quad t>0 .
$$

Suppose that for $t_{j}=j \Delta t, j=1, \ldots, N$, the value of $V_{t_{j}}$ is $v_{j}$. For simplicity, we assume that $\Delta t=1$. An unbiased estimate of $U\left(t_{j}\right)$ is given by

$$
u_{j}=\frac{1}{j(N-j-1)} \sum_{i=1}^{N-j}\left(v_{i+j}-v_{i}-m_{j}\right)^{2} \text {, }
$$




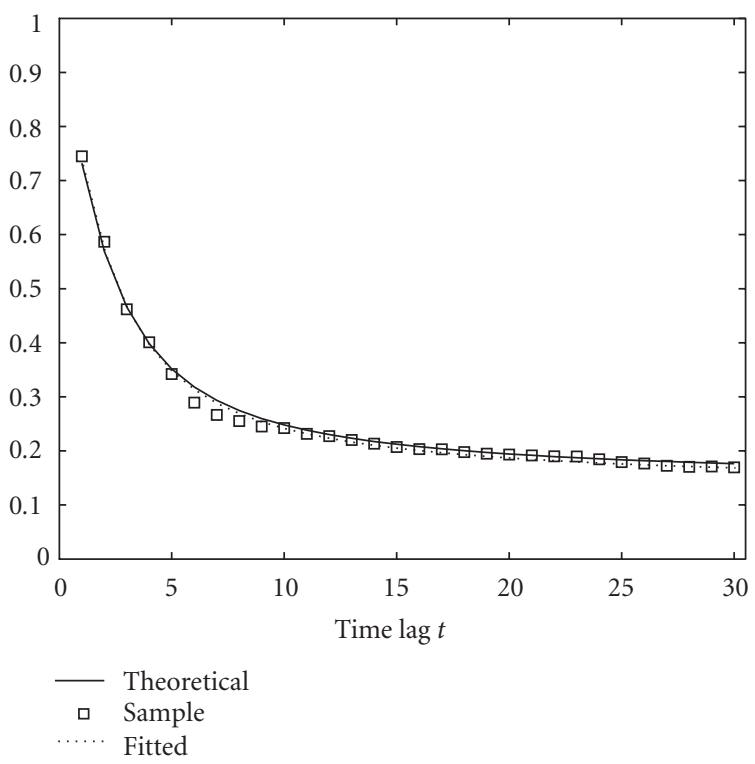

Figure 5.1. Plotting of the function $v(t)$, the sample data, and the fitted function $v_{0}(t)$.

where $m_{j}$ is the mean of $v_{i+j}-v_{i}$ 's:

$$
m_{j}=\frac{1}{N-j} \sum_{i=1}^{N-j}\left(v_{i+j}-v_{i}\right) .
$$

Fitting $\left\{U\left(t_{j} ; p, q\right)\right\}$ to $\left\{u_{j}\right\}$ by least squares, we obtain the desired estimated values of $p$ and $q$.

For example, we generate a sample $\left\{v_{1}, v_{2}, \ldots, v_{1000}\right\}$ for $V$ with $(p, q)=(0.5,0.3)$ by a Monte Carlo simulation. We use this data to numerically calculate the values $p_{0}$ and $q_{0}$ of $p$ and $q$, respectively, that minimize

$$
\sum_{j=1}^{30}\left(U\left(t_{j} ; p, q\right)-u_{j}\right)^{2} .
$$

It turns out that $p_{0}=0.5049$ and $q_{0}=0.2915$. In Figure 5.1 , we plot $\left\{U\left(t_{j} ; p, q\right)\right\}$ (theoretical), $\left\{u_{j}\right\}$ (sample), and $\left\{U\left(t_{j} ; p_{0}, q_{0}\right)\right\}$ (fitted). It is seen that the fitted curve follows the theoretical curve very well.

We extend the approach above to that for the estimation of the parameters $p, q, \theta$, and $\sigma$ in

$$
d X_{t}=-\theta X_{t} d t+\sigma d V_{t}, \quad 0 \leq t \leq T, X_{0}=\rho,
$$

where $\theta, \sigma \in(0, \infty)$, the process $V=\left(V_{t}, t \in[0, T]\right)$ is given by $(1.1)$ as above, and the initial value $\rho$ is independent of $V$ and satisfies $\mathbb{E}\left[\rho^{2}\right]<\infty$. The solution $X=\left(X_{t}, t \in\right.$ 
$[0, T])$ is the following Ornstein-Uhlenbeck-type process with memory:

$$
X_{t}=e^{-\theta t} \rho+\int_{0}^{t} e^{-\theta(t-u)} d V_{u}, \quad 0 \leq t \leq T .
$$

Put

$$
\varphi(t):=\int_{0}^{t} e^{(\theta-p-q) u} d u, \quad 0 \leq t \leq T .
$$

Proposition 5.1. It holds that

$$
\frac{1}{t-s} \mathbb{E}\left[\left(X_{t}-e^{-\theta(t-s)} X_{s}\right)^{2}\right]=H(t-s), \quad 0 \leq s<t \leq T,
$$

where, for $0<t \leq T$, the function $H(t)=H(t ; p, q, \theta, \sigma)$ is given by

$$
H(t)=\sigma^{2}\left\{1-\frac{p(2 q+p)}{(p+q)(\theta+p+q)}\right\} \frac{1-e^{-2 \theta t}}{2 \theta t}+\frac{\sigma^{2} p(2 q+p) e^{-2 \theta t} \varphi(t)}{(p+q)(\theta+p+q) t} .
$$

Proof. By (5.7), the left-hand side of (5.9) is equal to

$$
\frac{\sigma^{2} e^{-2 \theta t}}{t-s} \mathbb{E}\left[\left(\int_{s}^{t} e^{\theta u} d V_{u}\right)^{2}\right]
$$

We put $c(u)=p e^{-(p+q) u} I_{(0, \infty)}(u)$ for $u \in \mathbb{R}$. By Anh et al. [2, Proposition 3.2], $\int_{s}^{t} e^{\theta u} d V_{u}$ is given by

$$
\begin{array}{rl}
\int_{s}^{t} e^{\theta u} & d W_{u}-\int_{-\infty}^{\infty}\left(\int_{s}^{t} e^{\theta r} c(r-u) d r\right) d W_{u} \\
& =\int_{s}^{t}\left(e^{\theta u}-\int_{u}^{t} e^{\theta r} c(r-u) d r\right) d W_{u}-\int_{-\infty}^{s}\left(\int_{s}^{t} e^{\theta r} c(r-u) d r\right) d W_{u} .
\end{array}
$$

Thus $\mathbb{E}\left[\left(\int_{s}^{t} e^{\theta u} d V_{u}\right)^{2}\right]$ is equal to

$$
\int_{s}^{t}\left(e^{2 \theta u}-\int_{u}^{t} e^{\theta r} c(r-u) d r\right)^{2} d u+\int_{-\infty}^{s}\left(\int_{s}^{t} e^{\theta r} c(r-u) d r\right)^{2} d u
$$

By integration by parts and the equalities

$$
\begin{gathered}
\int_{u}^{t} e^{\theta r} c(r-u) d r=p e^{(p+q) u}\{\varphi(t)-\varphi(u)\}, \\
e^{-(\theta-p-q) s}\{\varphi(t)-\varphi(s)\}=\varphi(t-s),
\end{gathered}
$$

we obtain the desired result.

Suppose that for $t_{j}=j \Delta t, j=1, \ldots, N$, the value of $X_{t_{j}}$ is $x_{j}$. We assume that $\Delta t=1$ for simplicity. The estimation $h_{j}(\theta)$ that corresponds to $H\left(t_{j} ; p, q, \theta, \sigma\right)$ is given by

$$
h_{j}(\theta)=\frac{1}{j(N-j-1)} \sum_{i=1}^{N-j}\left(x_{i+j}-e^{-\theta j} x_{i}-m_{j}(\theta)\right)^{2},
$$


A. Inoue et al. 21

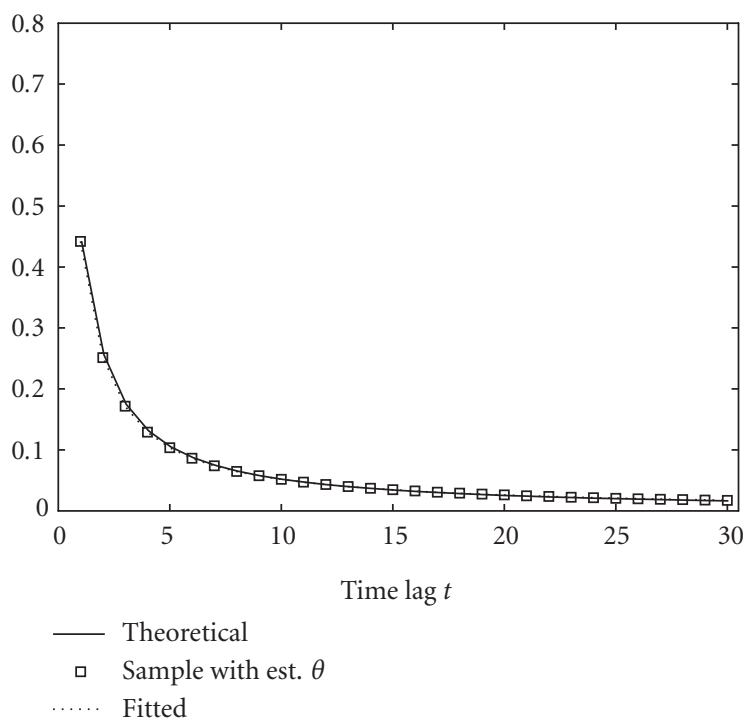

Figure 5.2. Plotting of the function $h(t)$, the sample data $\bar{h}(t)$ with estimated $\theta$, and the fitted function $h_{0}(t)$.

where $m_{j}(\theta)$ is the mean of $x_{i+j}-e^{-\theta j} x_{i}, i=1, \ldots, N-j$ :

$$
m_{j}(\theta)=\frac{1}{N-j} \sum_{i=1}^{N-j}\left(x_{i+j}-e^{-\theta j} x_{i}\right) .
$$

Fitting $\left\{H\left(t_{j} ; p, q, \theta, \sigma\right)-h_{j}(\theta)\right\}$ to $\{0\}$ by least squares, we obtain the desired estimated values of $p, q, \theta$, and $\sigma$.

For example, we produce sample values $x_{1}, x_{2}, \ldots, x_{1000}$ for $X$ with

$$
(p, q, \theta, \sigma)=(0.2,1.5,0.8,1.0)
$$

by a Monte Carlo simulation. We use this data to numerically calculate the values $p_{0}, q_{0}$, $\theta_{0}$, and $\sigma_{0}$ of $p, q, \theta$, and $\sigma$, respectively, that minimize

$$
\sum_{j=1}^{30}\left(H\left(t_{j} ; p, q, \theta, \sigma\right)-h_{j}(\theta)\right)^{2} .
$$

It turns out that

$$
\left(p_{0}, q_{0}, \theta_{0}, \sigma_{0}\right)=(0.1910,1.5382,0.8354,1.0184)
$$

In Figure 5.2, we plot $\left\{H\left(t_{j} ; p, q, \theta, \sigma\right)\right\}$ (theoretical), $\left\{h_{j}\left(\theta_{0}\right)\right\}$ (sample with estimated $\theta)$, and $\left\{H\left(t_{j} ; p_{0}, q_{0}, \theta_{0}, \sigma_{0}\right)\right\}$ (fitted). It is seen that the fitted curve follows closely the theoretical curve. 


\section{Simulation}

As we have seen, the process $V=\left(V_{t}, t \in[0, T]\right)$ described by (1.1) has both stationary increments and a simple semimartingale representation as Brownian motion does, and it reduces to Brownian motion when $p=0$. In this sense, we may see $V$ as a generalized Brownian motion. Since $V$ is non-Markovian unless $p=0$, we have now a wide choice for designing models driven by either white or colored noise.

In this section, we compare the performance of the optimal filter with the KalmanBucy filter in the presence of colored noise. We consider the partially observable process $\left(\left(X_{t}, Y_{t}\right), t \in[0, T]\right)$ governed by (1.6) with $\rho=0$. Suppose that an engineer uses the conventional Markovian model

$$
d Z_{t}=\theta Z_{t} d t+\sigma d B_{t}^{(1)}, \quad 0 \leq t \leq T, Z_{0}=0
$$

to describe the non-Markovian system process $X=\left(X_{t}, t \in[0, T]\right)$. Then, he will be led to use the Kalman-Bucy filter $\tilde{X}=\left(\tilde{X}_{t}, t \in[0, T]\right)$ governed by

$$
d \tilde{X}_{t}=\left(\theta-\mu^{2} \gamma(t)\right) \tilde{X}_{t} d t+\mu \gamma(t) d Y_{t}, \quad 0 \leq t \leq T, \tilde{X}_{0}=0
$$

where $\gamma(\cdot)$ is the solution to

$$
\frac{d \gamma(t)}{d t}=\sigma^{2}+2 \theta \gamma(t)-\mu^{2} \gamma(t)^{2}, \quad 0 \leq t \leq T, \gamma(0)=0
$$

(see (3.31) and (3.32)), instead of the right optimal filter $\hat{X}=\left(\hat{X}_{t}, t \in[0, T]\right)$ as described by Theorem 3.5 .

We adopt the following parameters:

$$
\begin{gathered}
T=10, \quad \Delta t=0.01, \quad N=\frac{T}{\Delta t}=1000, \\
t_{i}=i \Delta t \quad(i=1, \ldots, N), \\
\sigma=1, \quad \theta=-2, \quad \mu=5 .
\end{gathered}
$$

Let $n \in\{1,2, \ldots, 100\}$. For the $n$th run of Monte Carlo simulation, we sample $x^{(n)}\left(t_{1}\right), \ldots$, $x^{(n)}\left(t_{N}\right)$ for $X_{t_{1}}, \ldots, X_{t_{N}}$, respectively. Let $\tilde{x}^{(n)}(\cdot)$ and $\hat{x}^{(n)}(\cdot), n=1, \ldots, 100$, be the KalmanBucy filter and the optimal filter, respectively. For $u^{(n)}=\hat{x}^{(n)}$ or $\tilde{x}^{(n)}$, we consider the $a v$ erage error norm

$$
\mathrm{AEN}:=\sqrt{\frac{1}{100 N} \sum_{i=1}^{N} \sum_{n=1}^{100}\left(x^{(n)}\left(t_{i}\right)-u^{(n)}\left(t_{i}\right)\right)^{2}}
$$

and the average error

$$
\mathrm{AE}\left(t_{i}\right):=\sqrt{\frac{1}{100} \sum_{n=1}^{100}\left(x^{(n)}\left(t_{i}\right)-u^{(n)}\left(t_{i}\right)\right)^{2}}, \quad i=1, \ldots, N
$$


Table 6.1. Comparison of AEN's.

\begin{tabular}{l|cc}
\hline$\Theta$ & Optimal filter & Kalman-Bucy filter \\
\hline$\Theta_{1}$ & 0.5663 & 0.5667 \\
$\Theta_{2}$ & 0.4620 & 0.5756 \\
$\Theta_{3}$ & 0.5136 & 0.5167 \\
$\Theta_{4}$ & 0.4487 & 0.5196 \\
$\Theta_{5}$ & 0.4294 & 0.4524 \\
\hline
\end{tabular}

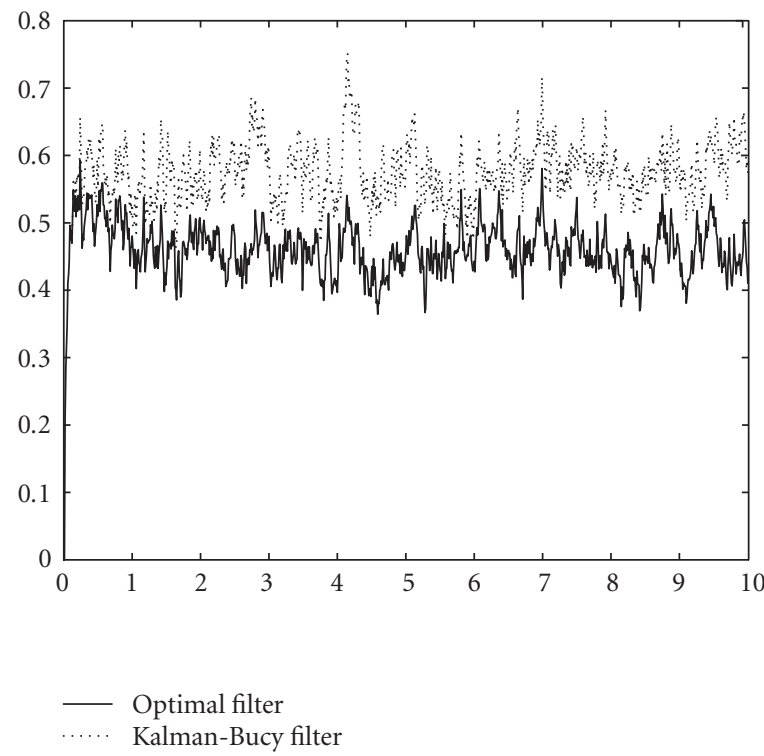

Figure 6.1. Plotting of $\mathrm{AE}(\cdot)$ for the optimal and Kalman-Bucy filters with noise parameter $\Theta=\Theta_{2}$.

In Table 6.1, we show the resulting AEN's of $\left\{\hat{x}^{(n)}\right\}$ and $\left\{\tilde{x}^{(n)}\right\}$ for the following five sets of $\Theta=\left(p_{1}, q_{1}, p_{2}, q_{2}\right)$ :

$$
\begin{aligned}
& \Theta_{1}=(0.2,0.3,0.5,0.2), \\
& \Theta_{2}=(5.2,0.3,-0.5,0.6), \\
& \Theta_{3}=(0.0,1.0,5.8,0.7), \\
& \Theta_{4}=(5.4,0.8,0.0,1.0), \\
& \Theta_{5}=(5.1,2.3,4.9,1.3),
\end{aligned}
$$

We see that there are clear differences between the two filters in the cases $\Theta_{2}$ and $\Theta_{4}$. We notice that, in these two cases, $p_{1}$ is large than the parameters $p_{2}$ and $q_{2}$. In Figure 6.1, we compare the graphs of $\operatorname{AE}(\cdot)$ for the two filters in the case $\Theta=\Theta_{2}$. It is seen that the error of the optimal filter is consistently smaller than that of the Kalman-Bucy filter. 


\section{Possible extension}

The two-parameter family of processes $V$ described by (1.1) are those with short memory. A natural problem is to extend the results in the present paper to a more general setting where, as in [1], the driving noise process $V=\left(V_{t}, t \in \mathbb{R}\right)$ is a continuous Gaussian process with stationary increments satisfying $V_{0}=0$ and one of the following continuous-time $\operatorname{AR}(\infty)$-type equations:

$$
\frac{d V_{t}}{d t}+\int_{-\infty}^{t} a(t-s) \frac{d V_{s}}{d s} d s=\frac{d W_{t}}{d t}, \quad t \in \mathbb{R}
$$

or

$$
\frac{d V_{t}}{d t}-\int_{-\infty}^{t} a(t-s) \frac{d V_{s}}{d s} d s=\frac{d W_{t}}{d t}, \quad t \in \mathbb{R}
$$

where $W=\left(W_{t}, t \in \mathbb{R}\right)$ is a Brownian motion and $d V_{t} / d t$ and $d W_{t} / d t$ are the derivatives of $V$ and $W$, respectively, in the random distribution sense. The kernel $a(\cdot)$ is a nonnegative decreasing function that satisfies some suitable conditions. More precisely, using the notation of Anh and Inoue [1], we assume that $a(\cdot)$ satisfies (S2) for (7.1), or either (S1) or (L) for (7.2). The assumptions (S1) and (S2) correspond to the classes of $V$ with short memory, while (L) to the class of $V$ with long memory. The process $V$ has an $\mathrm{MA}(\infty)$-type representation of the form

$$
V_{t}=W_{t}-\int_{0}^{t}\left(\int_{-\infty}^{s} c(s-u) d W_{u}\right) d s, \quad t \in \mathbb{R},
$$

for $(7.1)$ or

$$
V_{t}=W_{t}+\int_{0}^{t}\left(\int_{-\infty}^{s} c(s-u) d W_{u}\right) d s, \quad t \in \mathbb{R},
$$

for (7.2). For example, if $a(t)=p e^{-q t}$ for $t>0$ with $p, q>0$ in (7.1), then the kernel $c(\cdot)$ in (7.3) is given by $c(t)=p e^{-(p+q) t}$ for $t>0$, and (7.3) reduces to (1.1) for these $p$ and $q$.

For the stationary increment process $V$ in the short memory case (7.1) with (S2) or (7.2) with (S1), we can still derive a representation of the form (2.2) with (2.6), in which the kernel $l(t, s)$ is given by an infinite series made up of $c(\cdot)$ and $a(\cdot)$. In the long memory case (7.2) with (L), it is also possible to derive the same type of representation for $V$ if we assume the additional condition

$$
a(t) \sim t^{-(p+1)} \ell(t) p, \quad t \longrightarrow \infty
$$

or

$$
c(t) \sim \frac{t^{-(1-p)}}{\ell(t)} \cdot \frac{\sin (p \pi)}{\pi}, \quad t \rightarrow \infty,
$$

where $0<p<1 / 2$ and $\ell(\cdot)$ is a slowly varying function at infinity. Notice that (7.5) and (7.6) are equivalent (see Anh and Inoue [1, Lemma 2.8]). It is expected that results analogous to those of the present paper hold, especially, for $V$ with long memory, using the representation (2.2) with (2.6) thus obtained. This work will be reported elsewhere. 


\section{Acknowledgments}

We are grateful to the referee for detailed comments and the suggestion to insert the new Section 7 as a conclusion. This work is partially supported by the Australian Research Council Grant DP0345577.

\section{References}

[1] V. V. Anh and A. Inoue, Financial markets with memory. I. Dynamic models, Stochastic Analysis and Applications 23 (2005), no. 2, 275-300.

[2] V. V. Anh, A. Inoue, and Y. Kasahara, Financial markets with memory. II. Innovation processes and expected utility maximization, Stochastic Analysis and Applications 23 (2005), no. 2, 301-328.

[3] V. V. Anh, A. Inoue, and C. Pesee, Incorporation of memory into the Black-Scholes-Merton theory and estimation of volatility, submitted. Available at http://www.math.hokudai.ac.jp/ inoue/.

[4] R. S. Bucy and P. D. Joseph, Filtering for Stochastic Processes with Applications to Guidance, Interscience Tracts in Pure and Applied Mathematics, no. 23, InterScience, New York, 1968.

[5] M. H. A. Davis, Linear Estimation and Stochastic Control, Chapman and Hall Mathematics Series, Chapman \& Hall, London, 1977.

[6] J. B. Detemple, Asset pricing in a production economy with incomplete information, The Journal of Finance 41 (1986), no. 2, 383-391.

[7] D. Feldman and M. U. Dothan, Equilibrium interest rates and multiperiod bonds in a partially observable economy, The Journal of Finance 41 (1986), no. 2, 369-382.

[8] G. Gennotte, Optimal portfolio choice under incomplete information, The Journal of Finance 41 (1986), no. 3, 733-746.

[9] G. Gripenberg, S.-O. Londen, and O. Staffans, Volterra Integral and Functional Equations, Encyclopedia of Mathematics and Its Applications, vol. 34, Cambridge University Press, Cambridge, 1990.

[10] A. Inoue, Y. Nakano, and V. V. Anh, Binary market models with memory, submitted. Available at http://www.math.hokudai.ac.jp/ inoue/.

[11] A. H. Jazwinski, Stochastic Processes and Filtering Theory, Academic Press, New York, 1970.

[12] R. E. Kalman, A new approach to linear filtering and prediction problems, Transactions of the ASME. Series D: Journal of Basic Engineering 82 (1960), 35-45.

[13] R. E. Kalman and R. S. Bucy, New results in linear filtering and prediction theory, Transactions of the ASME. Series D: Journal of Basic Engineering 83 (1961), 95-108.

[14] I. Karatzas and S. E. Shreve, Methods of Mathematical Finance, Applications of Mathematics, vol. 39, Springer, New York, 1998.

[15] I. Karatzas and X. Zhao, Bayesian adaptive portfolio optimization, Option Pricing, Interest Rates and Risk Management, Handb. Math. Finance, Cambridge University Press, Cambridge, 2001, pp. 632-669.

[16] M. L. Kleptsyna, P. E. Kloeden, and V. V. Anh, Linear filtering with fractional Brownian motion, Stochastic Analysis and Applications 16 (1998), no. 5, 907-914.

[17] M. L. Kleptsyna and A. Le Breton, Extension of the Kalman-Bucy filter to elementary linear systems with fractional Brownian noises, Statistical Inference for Stochastic Processes. An International Journal Devoted to Time Series Analysis and the Statistics of Continuous Time Processes and Dynamical Systems 5 (2002), no. 3, 249-271.

[18] M. L. Kleptsyna, A. Le Breton, and M.-C. Roubaud, General approach to filtering with fractional Brownian noises-application to linear systems, Stochastics and Stochastics Reports 71 (2000), no. 1-2, 119-140.

[19] P. Lakner, Utility maximization with partial information, Stochastic Processes and their Applications 56 (1995), no. 2, 247-273. 
[20] _ Optimal trading strategy for an investor: the case of partial information, Stochastic Processes and their Applications 76 (1998), no. 1, 77-97.

[21] R. S. Liptser and A. N. Shiryaev, Statistics of Random Processes. I. General Theory, 2nd ed., Applications of Mathematics, vol. 5, Springer, Berlin, 2001.

A. Inoue: Department of Mathematics, Faculty of Science, Hokkaido University, Sapporo 060-0810, Japan

E-mail address: inoue@math.sci.hokudai.ac.jp

Y. Nakano: Division of Mathematical Sciences for Social Systems, Graduate School of Engineering Science, Osaka University, Toyonaka 560-8531, Japan

E-mail address: y-nakano@sigmath.es.osaka-u.ac.jp

V. Anh: School of Mathematical Sciences, Queensland University of Technology, G.P.O. Box 2434, Brisbane, Queensland 4001, Australia

E-mail address: v.anh@fsc.qut.edu.au 


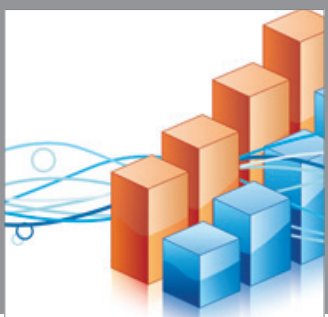

Advances in

Operations Research

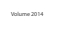

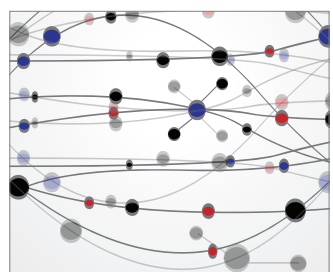

\section{The Scientific} World Journal
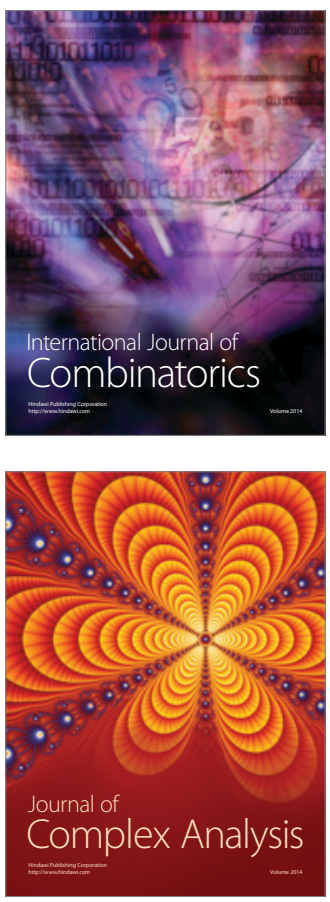

International Journal of

Mathematics and

Mathematical

Sciences
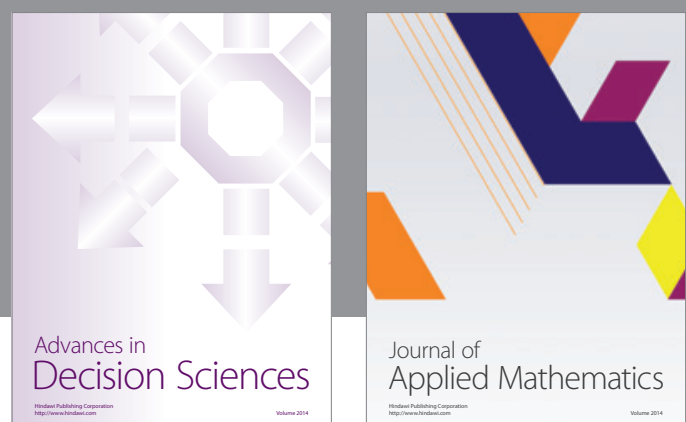

Journal of

Applied Mathematics
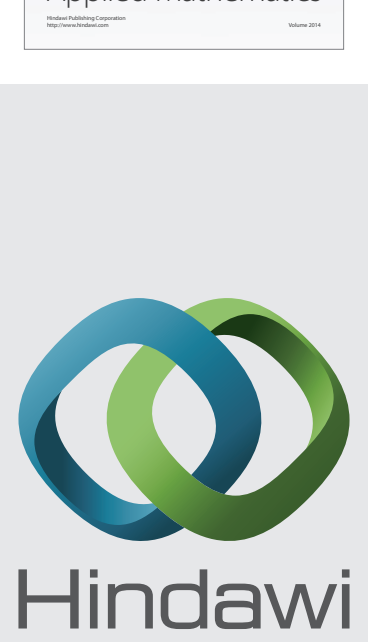

Submit your manuscripts at http://www.hindawi.com
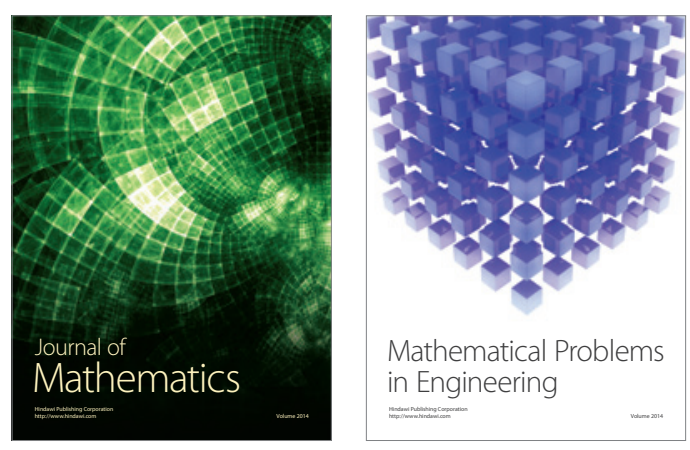

Mathematical Problems in Engineering
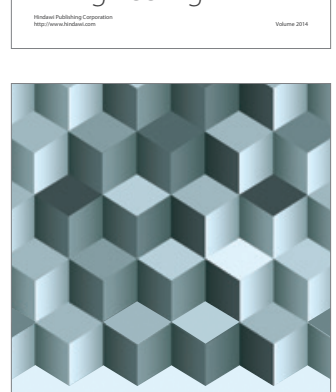

Journal of

Function Spaces
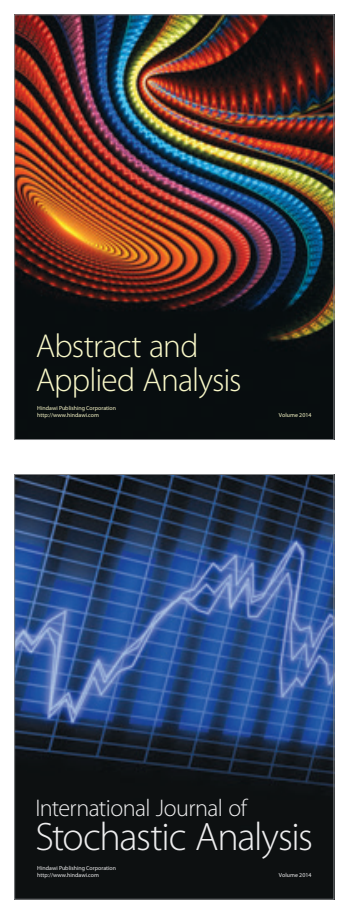

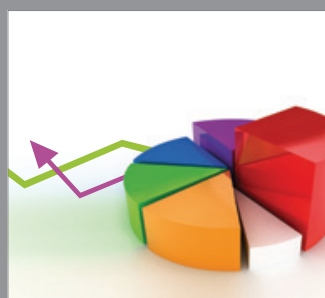

ournal of

Probability and Statistics

Promensencen
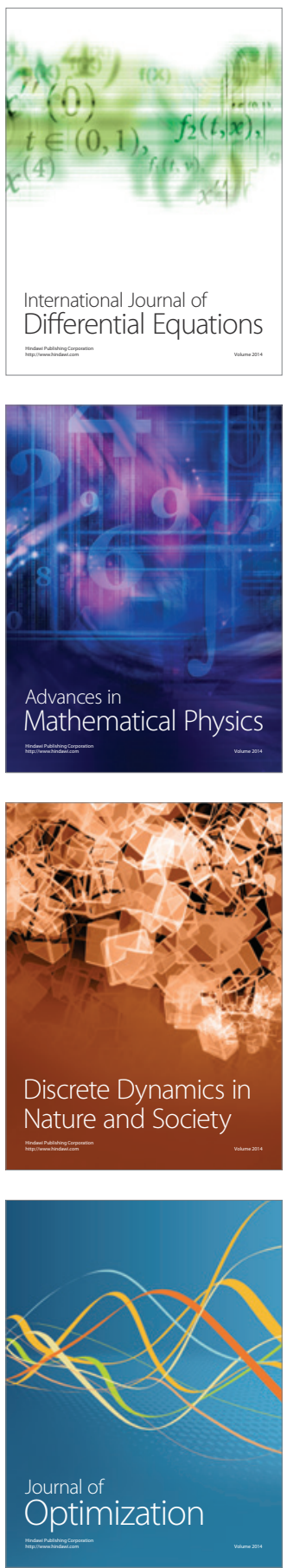